\section{Supervisory team:}

Dr C.A. Montgomery ${ }^{1}$

Dr. H. Poole ${ }^{1}$

Prof. H. Sumnall ${ }^{1}$

Dr. B. Frank ${ }^{2}$

${ }^{1}$ Liverpool John Moores

University, UK. ${ }^{2}$ The

Walton Centre,

Liverpool, UK

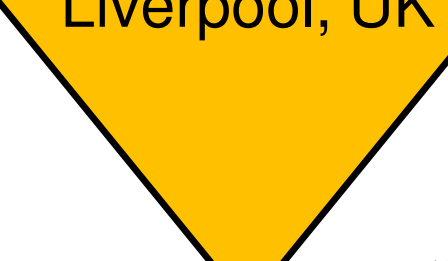

\section{INTRODUCTION AND AIMS}

There is little evidence to support long-term opioid medication for the treatment of Chronic Non-Cancer Pain (CNCP) [1]. Appropriately treating CNCP is a challenge for Health Care Professionals (HCPs) and patients who are prescribed opioids long-term are at increased risk of adverse effects such as tolerance and dependency [2-3]. There is a need to understand the lived experiences of opioid treatment from a patient and HCPs perspective. Understanding this further will provide insight to the many facets that encompass the course of the opioid treatment journey.

\section{AIMS}

The aim of this research is to establish a contextual understanding of what the treatment journey is like for both patient and HCP (i.e. what works well and not so well). In doing so, it will help to uncover common barriers and gaps within the opioid treatment journey. Findings will then be used as part of a wider piece of research developing an intervention for opioid weaning in primary care.

\section{METHODOLOGY}

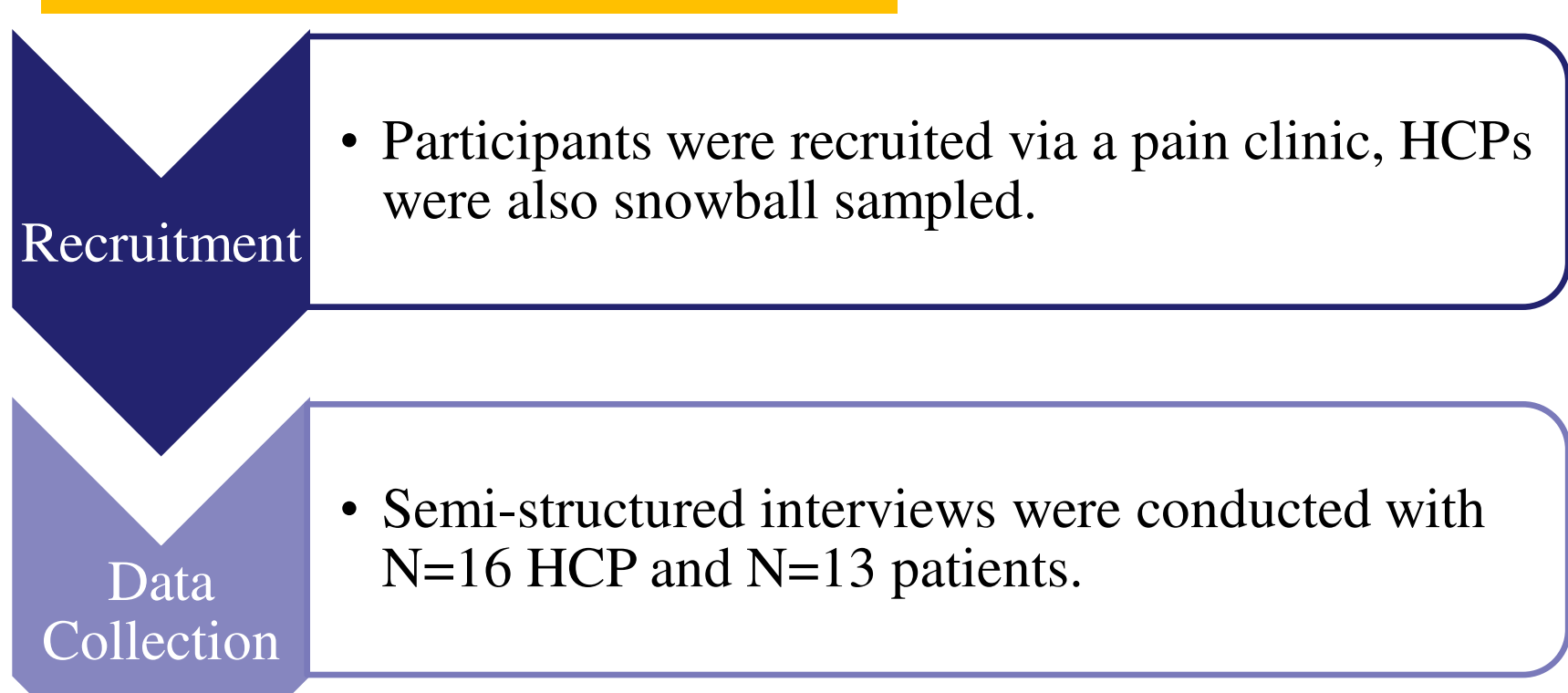

- An inductive thematic analysis was used to investigate this data.

\title{
3. FINDINGS
}

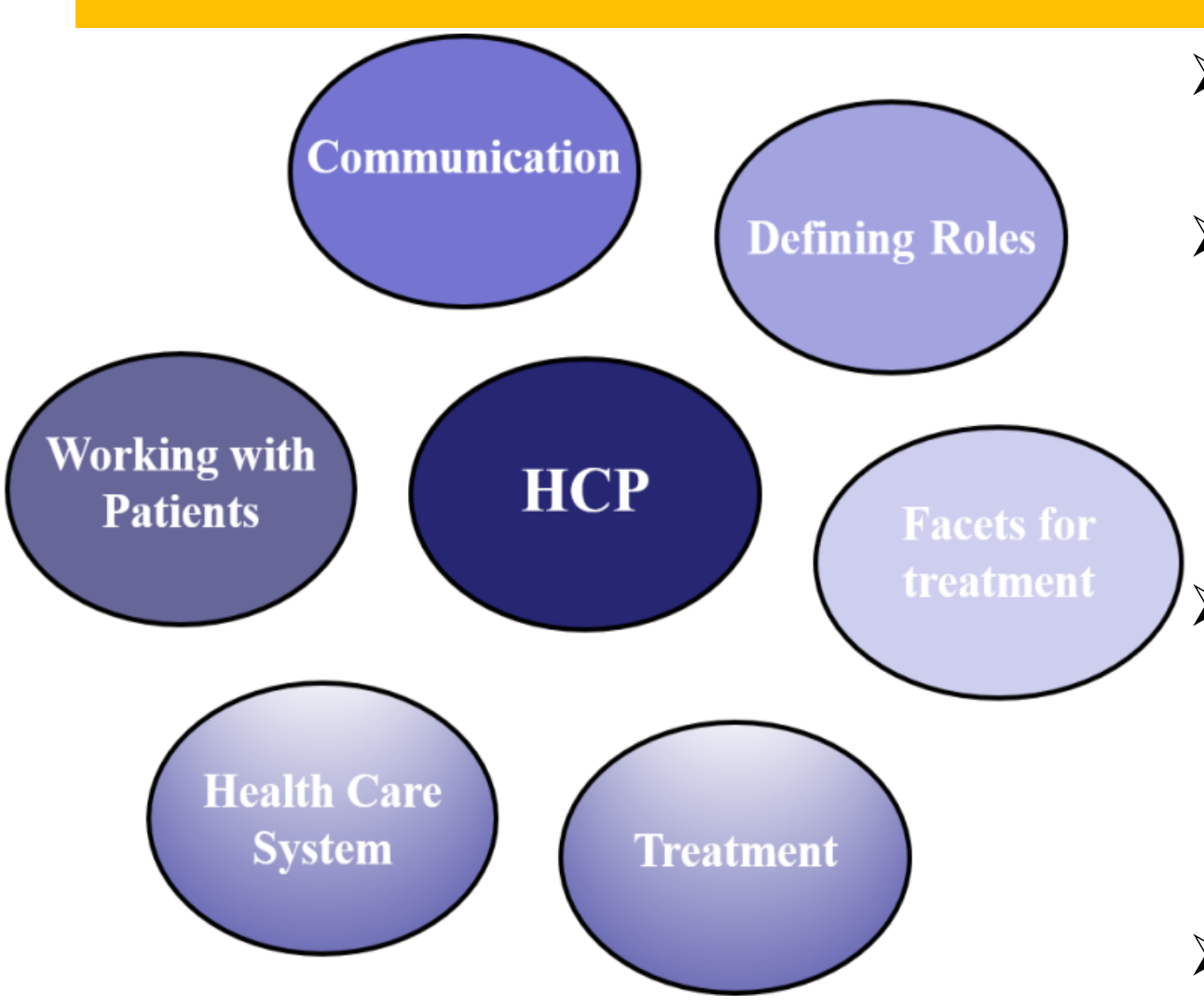

Generally patients discussed how they did not like taking their opioid medication due to the side effects, whilst also reporting "it's doing nothing for the pain" (P12). Regardless, they continued to take them to avoid withdrawal and as "a comfort thing" (P23).

$>$ Patients rarely visited their GP, unless for repeat prescription or a referral. They reported being prescribed opioids for years, with some also expressing thoughts that their General Practitioner (GP) wasn't knowledgeable enough in CNCP.

$>$ Patients commonly discussed needing more support during weaning, as they often struggled reducing their dose.

$>$ Most participants independently made the decision to come off their opioids.

"I'm doing it purely on my own, I would like some support"

$>$ There are six key themes that emerged from both the HCP and patient datasets.

$>$ HCPs often discussed a breakdown in communication when patients were treated across care sectors. This was made increasingly difficult when HCPs could not access appropriate Health Care Systems (HCS).

Limited time and capacity made it difficult treating CNCP patients, particularly when they often have complex conditions and "years worth of habit forming" (HCP11) behaviours that are difficult to undo.

$>$ Most HCPs believed opioids to be limited in their effectiveness at treating CNCP conditions and that there is a need to appropriately educate HCPs and patients.
"We don't have a combined system...I think that is where we are going wrong" (HCP10)

\section{"I don't want to be on these the rest of my life" (P23)}

"I haven't seen my GP in years" (P17) "I'm petrified of withdrawals" (P4)

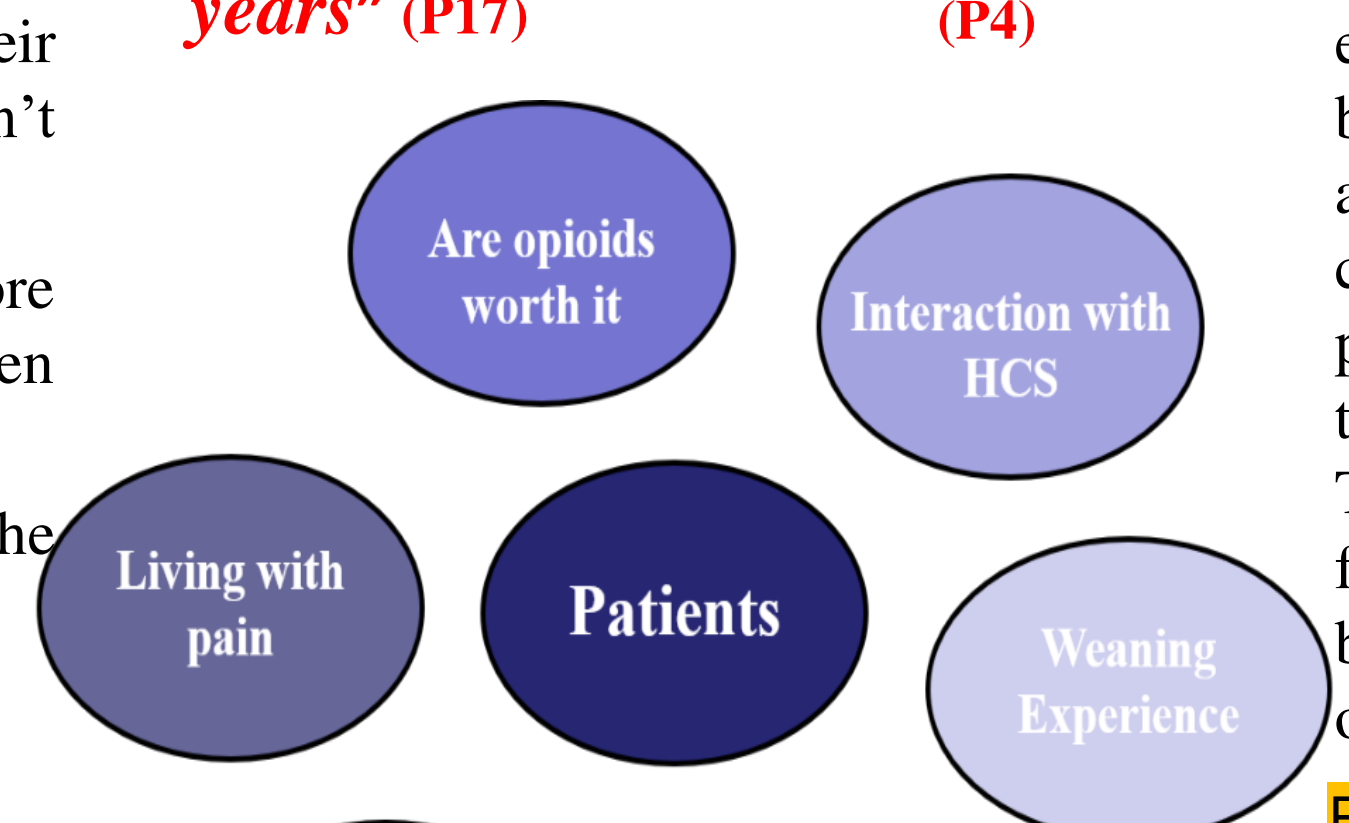

"We are doing them a disservice by not giving them other options, but we are limited in time and resources" (HCP26)

\section{"A lot of work needs to go into} patient education on opioids" (HCP13) 